\title{
Disaggregating the Asian "Other": Heterogeneity and Methodological Issues in Research on Asian Americans with Disabilities
}

\author{
Rooshey Hasnain ${ }^{1, *} \mathbb{C}$, Glenn T. Fujiura ${ }^{1, *}$, John E. Capua ${ }^{2, *}$, Tuyen Thi Thanh Bui ${ }^{3, *}$ \\ and Safiy Khan ${ }^{1, *}$ \\ 1 Department of Disability and Human Development, Institute on Disability and Human Development, \\ and Undergraduate Rehabilitation Sciences Program, University of Illinois at Chicago, Chicago, \\ IL 60608, USA \\ 2 New York-Presbyterian Hospital, New York, NY 10065, USA \\ 3 School of Social Work, University of Illinois at Urbana-Champaign, Urbana, IL 61801, USA \\ * Correspondence: roosheyh@uic.edu (R.H.); gfujiura@uic.edu (G.T.F.); jcapua2@gmail.com (J.E.C.); \\ ttbui2@illinois.edu (T.T.T.B.); skhan329@uic.edu (S.K.)
}

Received: 1 July 2020; Accepted: 21 July 2020; Published: 28 July 2020

\begin{abstract}
Asian Americans comprise the fastest growing racial or ethnic group in the US. Between 2000 and 2019, their numbers almost doubled, from 11.9 million to 22.2 million. The numbers of people with disabilities within this demographically important population, which are also growing, puts stress on the service delivery sector. This situation indicates a pressing need for research on lived experiences of disabled Asian Americans. A review of the extant literature shows that Asian Americans are underrepresented in the research on disability and/or mental health. This lack of hard data is compounded by the tendency to treat Asian ethnicities as monolithic. The US Census Bureau recognizes more than 20 distinct Asian nationalities, ranging from South Asian Pakistani Americans to Southeast Asian Americans. Aggregating all Asian Americans together in surveys and studies impedes a sophisticated understanding of their unique needs and strengths. From a policy or systems perspective, inadequate data representation in the research literature, including outdated conclusions, is an implicit form of disenfranchisement. This conceptual article examines issues and implications around the lack of systematic attention to diversity within the Asian American population in disability research.
\end{abstract}

Keywords: research methods; disparity; disaggregating data; Asian Americans; disability; mental health; model minority myth

\section{Introduction}

The Asian American population is one of the fastest-growing racial groups in the United States [1]; within this heterogenous group, many are living with disability or caring for a family member with a disability. Historically, however, Asian Americans, including Asian Americans with disabilities and/or mental health conditions (hereafter 'disability,' as reported by Article 1 of the Convention on the Rights of Persons with Disabilities [2], have been underrepresented and/or misrepresented in US-based research studies [3]. This lack of attention is compounded by the tendency of disability-related studies to target middle-class Whites (i.e., European Americans), and occasionally African Americans, Latinos, and Native Americans, in discussions of theories, policies, programs, and practices in the relevant disciplines. As a result, little research has focused on the needs and strengths of an important and growing Asian demographic in the context of disability rights and advocacy [4]. 
From a systems or policy perspective, inadequate data representation in the research literature, including outdated conclusions, is an implicit form of disenfranchisement. The purpose of this conceptual article is to examine broader implications of this lack of systematic attention to diversity within the Asian American population in disability research. This article also presents alternative methodologies that can help researchers better address the heterogeneity of Asian Americans in responsive and inclusive ways. In the sections that follow, we introduce underlying demographics and discuss approaches to expanding the research relating to Asian Americans with disabilities.

\subsection{Demographics and Approaches to Expanding Research}

Demographically, this article uses the United Nations description of Asia as including more than 4.1 billion people, living in 51 countries, dependencies, or territories, with hundreds of spoken languages, and many faiths [5]. Failure to account for such diversity can have potential consequences if research lacks the necessary sensitivity to identify important differences within the broader Asian American population. In the US, as elsewhere, government policy is often shaped by such studies. Moreover, the implementation of culturally tailored outreach across different service sectors, including disability-related supports, is based on perceived demographic and cultural characteristics. Some of these may be outdated or inaccurate if they are based on research whose premises are flawed; therefore, our understanding of how these services affect Asian Americans' quality of life (e.g., differences in lack of access to information or resources or variations in participation rates) may be limited [6].

According to the 2017 estimate by the US Census Bureau, Asian Americans comprise 22.2 million people, about $6 \%$ of the total US population [7]; by 2050, this number is expected to increase to 41 million, totaling $9 \%$ of the US population [8]. The growing size and diversity of the Asian American population presents good opportunities to expand research on issues of access, participation, and well-being. Participatory methods and approaches to research [9] for pan-Asian communities can fill the present gap and by doing so contribute to providing a better quality of life for Asian Americans living with disability.

As one might expect, the diversity of Asia is largely reflected in the demographics of Asian Americans. For example, the Pew Research Center [10], and Ramakrishnan and Ahmad [11] have identified more than 38 spoken languages, more than 38 ethnic designations, and more than 8 religious belief systems, along with 40 countries of origin. Information shared in advance of the 2020 Census at the VietAID hearing [12] raises other important considerations that are relevant to conducting local or state research on this vastly heterogenous group:

"Most cities and counties in Massachusetts do not have large enough populations to enable this kind of analysis using federal data collections, such as the American Community Survey collected by the U.S. Census Bureau. State and local data collections of detailed Asian origin are critical to fill these gaps, and to help formulate and implement policies that benefit local populations. It is also critical that such data be collected and disseminated in a manner that protects individual privacy and ensures data security, consistent with safeguards on other types of state and local data collections."

(Ramakrishnan, Director, AAPI Data; Professor of Public Policy).

Asian American diversity, of course, is also reflected within the demographics of those who have disabilities. If support intervention and rehabilitation strategies are to be optimized, policy makers, as well as researchers and service delivery personnel, must understand and accept the importance of underscoring this diversity in ways that are both accurate and up-to-date. It is urgent that the design and implementation of culturally appropriate outreach and service delivery rest on reliable demographic information.

If they are to conduct culturally responsible studies, researchers must understand their own biases, values, and cultures [9]. It is also necessary, when seeking to address service delivery and policies that can be adapted to cover all Asian cultural standards and beliefs, to acknowledge core differences in 
research styles between the East and West. In Eastern culture, respected community elders may share stories with younger generations. Traditionally, specialized knowledge, such as methods of coping with or acknowledging an impairment or disability, is not shared openly; only these "gatekeepers" are trusted with holding and imparting such knowledge. This culturally-based, time-honored restriction can present conflicts with the typical Western research paradigm, which emphasizes academically-derived knowledge that is openly shared across chronological ages [4].

\subsection{Asian Diversity Is Oversimplified}

Despite their increasing presence and visibility in the U.S., Asian Americans—on individual, family, and group levels-are overlooked and ignored; their diversity is oversimplified, and they are treated as a homogeneous entity in research, especially in studies focused on disabilities [13,14]. Because of these problems, Asians with disabilities living in the US, whether they are foreign-born immigrants, refugees, or native-born citizens, remain underserved, misunderstood, and under-researched both in their own communities and within the American mainstream [15]. The limited available literature implies that this lack of information has consequences that are both serious and wide-ranging [16]. According to Ngo-Metzger, Kaplan, Sorkin, Clarridge, and Phillips [17], Asian Americans have more problems with specialized services than Whites, and tended to be dissatisfied with the service they received; we may speculate that these conditions hold true for disability services, as well [3]. Moreover, the presence of cultural gaps between clients and providers makes it difficult for researchers to craft intervention solutions within a given setting, while also upholding their research principles and methodologies [18-21]. A review of the scant literature on this topic clearly indicates a lack of adequate attention to disparities in disability services or supports overall. It also indicates the disregard of the research contributions of Asian-serving community-based organizations (CBOs), especially in Asian American sub-ethnic communities [22].

Inadequate data likely make it easier for state and federal programs in the disability and rehabilitation service sectors either to overlook the needs of Asian Americans or fail to establish benchmarks for services and outcomes [23]. In addition, traditional stigmas associated with disability within Asian American communities themselves, combined with other cultural factors, may conceal not only the immediate needs of Asian Americans with disabilities but also their human potential. As a result, disabled Asians often remain invisible within their own communities as well as to the "outside world" [16]. In addition, researchers working to improve access and opportunities for and with Asian Americans with disabilities are still struggling to find culturally and linguistically competent ways to conduct useful and responsible research [6].

White House Executive Orders 13125 and 13515, both issued in the late 1990s, recognized the need to increase participation and to improve quality of life among Asian Americans and Pacific Islanders who were deemed to be underserved by federal programs.) [24,25]. Our motivations in reviewing research challenges and publicizing alternative strategies are to increase the knowledge base around a significant US demographic underserved by disability services and supports; to help this demographic overcome a variety of complex, daunting challenges; and to refute pervasive myths and stereotypes that contribute to the research gap. In the next section, we introduce the issues pertaining to the under-representation and misrepresentation of Asian Americans with disabilities.

\subsection{The Research Dilemma: Heterogeneity of Asian Americans and the Model Minority Stereotype}

Failure to recognize the heterogeneity of Asian Americans in research perpetuates the "model minority" stereotype [26] - a problematic tendency that, by assigning homogeneity to the Asian American community, not only undermines its complexity and diversity but also disregards significant difficulties encountered by its less-privileged subgroups. In addition, as previously discussed, the category "Asian American" in itself is an aggregate that masks significant differences [27]. The reality, as Chung found in 1995, is that Asian Americans are too often lumped together as a single group in quantitative studies; similarly, in qualitative studies, individual groups are considered representative 
of all Asian Americans [28]. To illustrate, a study by the Pew Research Center [29] examined Asian Americans' socioeconomic environment and quality of life and found this group to be the highest achieving racial/ethnic group in terms of educational attainment and economic status. However, although the article claims that Asian Americans have the highest income among racial/ethnic minority groups (a median annual income of $\$ 66,000$ compared to the national median of $\$ 49,000$ ), a more thorough analysis of the economic status of Asian American subgroups presents a different picture. Citing data from the 2010 Census, Hing [30] reports that poverty rates among Cambodian and Laotian Americans are as high as that of African Americans. Thus, by lumping Asian Americans into one category, and using median salary across racial/ethnic minorities as a marker, the Pew study seems to suggest that Asian Americans are bypassing economic challenges. Yet these challenges are clearly at issue for certain Asian American subgroups.

The failure to acknowledge Asian American heterogeneity not only ignores economic disparities between Asian American subgroups, which contributes to the marginalization of some subgroups in research and public policy, but also ignores differences in their academic achievement. Stereotypically, Asians are thought to be academically successful because of their hardworking attitude and intelligence, but a 2016 study by Joo and colleagues [26] challenges the belief that Asian Americans do well in school because they attend better schools, a belief bolstered by observations that school performance of lower-income members of racial and/or ethnic groups is poorer by comparison. By calculating the average math passing rate of schools within each Public-Use Micordata Area (PUMA), without data from Asian American students to account for potential skew, the authors ranked PUMAs from best to worst. They found that Asian students in low-performing school districts fared as poorly as some of their African American counterparts in similar school districts. They also found wide disparity in school performance among Asian American subgroups. For example, in California (where over $30 \%$ of Asian Americans reside), the difference between the mean school quality percentile of the highest-performing Asian American subgroup, Taiwanese Americans, and the lowest-performing subgroup, Hmong Americans, was around 30 percentage points.

As both Hing [30] and Joo et al. [26] point out, the assumption that Asian Americans constitute a monolithic group renders differences within and between subgroups invisible. This practice also risks perpetuating the flawed assumption that Asian Americans are completely self-reliant and prosperous. Consequently, the discourse around the model minority stereotypes does grave injustice to less advantaged subgroups, such as Burmese Americans, whose unique needs may not be adequately represented in federal, state, and local policies. In the next section, we introduce the issues pertaining to underrepresentation and misrepresentation of Asian Americans with disabilities.

\section{The Under-Representation and Misrepresentation of Asians Americans with Disabilities in Research}

Despite the growing need for accurate information about Asian Americans with disabilities, researchers have not significantly added to the literature on their lived experiences. This dearth of information is particularly relevant in the contexts of disability and rehabilitation service access and outcomes [20,31]. The few existing empirical studies of the status of Asian Americans in the disability sector affirm that this population is underserved and receives lower-quality support and rehabilitation compared to other groups, including Latinos and Blacks/African Americans [32]. Sample biases would suggest the problem is greater than reported, as such data are based only on individuals who seek and obtain disability and rehabilitation services. Cultural reticence of those who need services but choose not to obtain them appear greater among Asian American subgroups [33]. Researchers across numerous disciplines agree, however, that incorporating diversity into representative samples is neither simple nor straightforward [33].

In short, the lack of disability data meaningfully disaggregated within the larger Asian American population leads to erroneous assumptions, such as: (1) this population can be categorized as a single group; (2) subgroups have homogeneous needs for disability and rehabilitation supports; 
and (3) intra-group variations are irrelevant inputs to improvements in service access and delivery, both on pragmatic and policy levels. Given the reported rates of disability by Asian Americans, these assumptions are understandable. However, it cannot be overemphasized that they are likely inaccurate and are potentially misleading due to intra-group variations and undersampling [29]. According to the American Community Survey 2017, the reported rate of disability among Asian Americans was $4.4 \%$. This percentage, which was remarkably low to begin with, was also the lowest among all racial categories. The 2017 Disability Status Report found the following numbers for other racial groups: Whites, 10.6\%; Blacks, 13.6\%; and Native Americans, 18.1\%; and other races, 9.5\% [34].

Chung [28], who explored health and disability rates among Asian Americans in Massachusetts, asked whether Asian Americans are a healthy minority; an underserved, hard-to-reach population with health and disability needs similar to other ethnic groups; or both. Results indicated that Asian Americans with disabilities are an underserved population due to both limited research and the lack of individual narratives. Unfortunately, answers to these and similar disability-related questions may be difficult to find due to "inadequate resources to support racially and ethnically disaggregated Asian American research and data collection activities with appropriate linguistic and cultural translations" [35] (p. 12). In the next section, we introduce our structured literature search and examples of our findings.

\section{Literature Search: Research on Asian Americans with Disabilities}

Three original research authors conducted a structured literature search of published studies in order to summarize and evaluate the existing research methodologies used to explore disability topics within Asian American communities. We reviewed original research studies if (1) they were conducted in the US or in English-speaking countries (e.g., the UK), if (2) Asian Americans (either as a "whole community" or as individual ethnic groups) were a population of interest, and if (3) disability was the central focus of the research. Search terms were "Asian American" and "disability." After this search was complete, using EBSCO, PubMed, and PsychInfo, the two lead authors reviewed the results.

Our selection process was deliberately over-inclusive so we could retrieve as much literature as possible. We considered a range of disabilities, both physical and mental. To merit inclusion, articles had to meet the following requirements, in addition to the three parameters enumerated above. First, they had to state how disability status was defined or assessed; disability indicators had to be used in large survey questionnaires: samples in any type of study had to include respondents who self-reported or self-identified as having an impairment or disabling conditions; and they had to include an explanation of the diagnosis of the disability, especially for young children with developmental disabilities. Studies that did not primarily focus on Asian Americans or that included other ethnic/racial groups (e.g., studies based on national surveys) were screened and reviewed only if they provided data collection methods for and analyses of Asian Americans. Although this paper is largely social science-oriented, we included articles that are primarily health-related if they contain methods directly pertaining to Asian Americans with disabilities, broadly defined.

Publications that merited inclusion originated from original, primary, peer-reviewed studies published between 2000 and 2020, regardless of the publication source. Our search sought articles published in English with full-text availability. We excluded articles lacking a section on "methods" or "analyses". We also excluded letters or editorials, abstracts from meetings and conferences, commentaries, and studies that did not have a central focus on disability. We reviewed each selected article with an eye to its "methods" and "analyses" sections.

Our analysis of the literature focused on the following factors: study designs; recruitment/data collection methods, including research settings; and, if applicable, the specific Asian American ethnic group(s) in question. After we satisfied these criteria, each article was then summarized and analyzed to assess emerging themes and patterns relevant to the methodologies used in disability research on Asian American populations. 


\section{Search Results}

Table 1 summarizes the research literature showing: (1) the absence of Asians in studies of minorities and (2) that studies tend to lump all Asians together or only focus on one subgroup (e.g., Chinese). From the 333 identified references, 59 studies met the inclusion criteria as noted in (Table 1). A total of 177 articles were initially screened by title. Of these, 87 abstracts were selected for review. We excluded 28 articles because they were not original. A total of 18 articles were reviewed from EBSCO, 23 from PubMed, and 18 from PsychInfo (see Supplementary Material 1).

Table 1. Demonstration of search results.

\begin{tabular}{cc}
\hline Search Activities & Number of Articles \\
\hline Records identified through database searches & 333 \\
Initial screening by title & 177 \\
Abstracts were selected for review & 87 \\
Non-original articles & 28 \\
Studies included in this paper & 59 \\
EBSCO & 18 \\
PubMed & 23 \\
PsyInfo & 18 \\
\hline
\end{tabular}

Most of the studies we included conducted quantitative analyses $(n=40)$. They ranged from community-based surveys to large national database-driven studies. Many of the latter, for example the American Community Survey and the National Health Interview Survey, included Asian Americans; another, De Souza and Fuller-Thomson [36], used the American Community Survey to examine self-reported disability outcomes of older Filipino Americans. Another [37] used the National Latino and Asian American Study dataset to conduct analyses on psychological outcomes among various Asian American ethnic groups with disabilities, where disability status was determined using a four-item assessment questionnaire.

Some of the statewide and community-based studies used original surveys developed for the purpose of studying disability among Asian Americans exclusively. For example, a few studies of Korean American elders with functional limitations and depressive symptoms [38-40] used a variety of sources for recruitment, including community-based organizations (CBOs), religious associations, and a telephone directory of residents. These studies assessed disability status using a variety of performance scales, such as the Personal Activities of Daily Living (PADL) and Instrumental Activities of Daily Living (IADL) questionnaires. One study that examined Asian American students with learning disabilities, from different ethnic groups [41], utilized data from a large suburban school district whose special education records were used to identify the broad cohort. Overall, these quantitative studies used either existing large datasets or developed original surveys to collect data on disability among Asian Americans. The studies that conducted Asian American-specific surveys sampled local communities and targeted ethnic-specific settings (e.g., businesses, associations, and churches) as the primary data sources.

Some of the studies used qualitative approaches $(n=12)$. Most were based on single interviews; one used focus groups. Two studies [42,43] used semi-structured interviews to collect self-reported data from Chinese American families with young children diagnosed with developmental disability, specifically autism, and utilized professional networks and public advertisement for recruitment. All of the qualitative studies used local Asian American communities as the principal source of participants. One study of South Asian Indians with children having developmental disabilities [44] utilized the author of the study's personal connections as the primary method of recruitment. Another study conducted focus groups and in-depth interviews among Hmong Americans with family members with developmental disability. 
Baker, Miller, Dang, Yaangh, and Hansen [45] recruited participants primarily through collaboration with local Hmong CBOs. One of the studies, by Kwong, Chung, Cheal, Chou, and Chen [46], used a mixed-methods design that included both survey data collection and in-depth interviews. This study was conducted at a US community health center that serves neighborhoods with high densities of Chinese families. The center recruited patients who screened positive for depression.

Overall, the studies on topics related to disability among Asian Americans as a community found were found to have a wide variety of data sources and collection processes. Quantitative studies were primarily based on large extant datasets (in which Asian Americans were routinely part of the data survey process) or smaller original surveys for which Asian Americans were exclusively the target population. The qualitative studies utilized specific local communities, targeting the Asian Americans within them.

The use of large non-Asian American-specific datasets is problematic because disability is reportedly a relatively low-prevalence condition among Asian Americans. In addition, Asian Americans typically comprise a very small percentage of the total disability sample. Small samples in representative surveys were found to present data reliability issues as well. Therefore, although large sophisticated surveys may include identifiers for multiple Asian ethnic groups, we could not disaggregate data from them because of inadequate sample sizes. Furthermore, we found that qualitative studies, despite providing the richness of contextual detail that quantitative studies are not designed to capture, tend to involve limited or single-person sample sizes [43,45]. Next, we present some of the challenges and limitations of conducting research on Asian Americans who are living with disability.

\section{Ignoring Asian Diversity}

In 2017, the Asian American population consisted of people from at least 29 Asian countries, speaking hundreds of languages and belonging to numerous religions. The majority of Asian Americans residing in the United States are first-generation immigrants; of these, $77 \%$ speak no or limited English [11,47]. The few extant studies on this population have tended to combine a broad range of Asian American subgroups (e.g., Chinese American, Pakistani American, Cambodian American, Korean American, and Japanese American) into a single category. This practice is misleading, conceals vital information, and has serious, real-world consequences. In terms of disability research and practice, this consistent reduction masks the range of disparities and outcomes within and across subgroups of Asian American people with disabilities, and across discrete Asian American populations.

As a group, Asian Americans report that $26.5 \%$ of families have at least one member with a disability [48]. This is the lowest reported disability rate; all other racial or ethnic sub-populations report higher rates: American Indian/Alaska Natives, 38.5\%; Blacks, 35.7\%; Latinos, 33.2\%; Native Hawaiian/Pacific Islanders, 31.6\%; and Whites, 27.5\%. The exact reasons for this difference, while unknown, are likely related to underreporting due to cultural stigma or sampling biases, restrictive immigration policies, differential risks, or some combination of these factors. It is critical for disability scholars and service providers to develop useful research strategies that effectively incorporate multicultural or indigenous theories into Asian ethnic studies (vs. non-Asian) on the service and intervention needs of Asian Americans with disabilities, their families, and their communities [49].

Another confounding factor, and one that contradicts the "healthy model minority" myth to the detriment of Asian Americans, is that researchers in the US tend to compare majority with non-majority individuals and families without considering their cultural contexts. As noted by Chin et al. [50], "Often these approaches have been used as a comparative approach where Asian Americans are compared with Whites as a norm, and in so doing Asian Americans are found to be deficient" (p. 118). Chin et al. [50] also observe that studies often describe Asian Americans as less verbal, dominant, and aggressive, as well as more "alienated" than Whites, absent a context-based elaboration. Fortunately, greater awareness of and emphasis upon cross-cultural and cross-ethnic perspectives has begun to encourage researchers to examine the actual cultural processes and functioning of Asian 
American families and communities. Unlike the deficit model, this approach emphasizes the strengths of inter- and intra-familial variations [6].

To illustrate, the Asian American Psychological Association Leadership Fellows Program [51] reported two alarming (Asian and non-Asian) findings: among all Asian Americans, those aged 20-24 had the highest suicide rate (12.44 per 100,000), and among older females from all racial backgrounds, between the ages of 65 and 84, Asian Americans ranked first among them for suicides. A related finding indicates that some groups of Asian Americans may suffer trauma-related illnesses at rates higher than average among other US-based ethnic and racial groups. Another study affirms these findings by showing prevalence rates of acute levels of depression and post-traumatic stress disorder as $68 \%$ for Cambodian refugees vs. $37 \%$ for Whites [22].

\subsection{Inconsistent Taxonomies}

In the studies that estimated disability among Asian American populations, variations in data collection strategies and inconsistent classifications compound an already confusing situation. For example, some sources report on "Asians" or "Asians and others," whereas others report on "Asians and Pacific Islanders." These issues can be traced to two modifications to the criteria for the Asian racial category made by the US Office of Management and Budget (OMB) in 1997. First, the "Native Hawaiians and Other Pacific Islander" category was separated from "Asian." Second, respondents could designate more than one racial/ethnic category. The Census Bureau's population estimates distinguish an Asian "alone" classification (only one racial or ethnic designation) from an "in-combination" classification (multiple racial or ethnic designations that include one Asian category). "These changes make it more difficult to identify a respondent's preferred value, or the correct value for a respondent" [1]. Moreover, available data on Asian American disability rates may not accurately reflect reality because of definitional differences and sampling procedures. The key issue is the lack of consensus regarding the most meaningful taxonomy for Asian racial and ethnic groupings.

\subsection{Sampling Limitations}

Even when samples are obtained from Asian American groups in healthcare- or social sciencerelated studies, there are often not enough respondents to derive statistically stable estimates. Similarly, in disability-related publications (identified in Supplementary Material 2), a matrix of 34 included articles using population data focused on minorities, but excluded Asians. For example, Akobirshoev et al. [52] focused only on Whites, Blacks, and Hispanics. Thus, despite the significance of the population, even disability and/or population-level studies that focused on racial and ethnic minority populations frequently excluded Asians of all types.

The disparities, inconsistencies, and design problems mentioned above deny potentially important findings on health, employment, and other topics that are of interest to researchers, service practitioners, and policy makers. As a result, we know little about the wide-ranging and complex cultural factors (e.g., differences associated with immigrant versus refugee status and also with health beliefs, customs, migration patterns, help-seeking behaviors, and religions) that influence Asian Americans with disabilities and their families [53]. For example, differences associated with immigrant versus refugee status, health beliefs, customs, migration patterns, help-seeking behaviors, and religions vary dramatically across subgroups and can differentially impact disability related issues.

Ghosh [14] states that "the vast majority of data for Asian Americans is listed as 'data not collected,' 'statistically unreliable,' or 'data not analyzed'" (p. 76). The lack of dedicated data may also perpetuate the model minority myth; namely, that Asian Americans are invariably healthy, intelligent, successful, and problem-free. In particular, some US-based researchers have considered Asian Americans "the healthy" or "the model" minority, based on the assumption that they routinely achieve higher levels of success than other groups and also inherently face fewer health risks due to the comparative advantages of protective factors, such as strong family ties, religiosity, and a deep sense of community [26]. Implicit in this portrayal is that the need for disability and related support 
services in this community is minimal, both because this population allegedly experiences lower rates of disability and because it is believed that family and community will adequately address the need when it appears [54].

In short, our grasp of the complexity created by variations across Asian American race/ethnicity, culture, language, immigration status, acculturation with respect to disability, is extraordinarily limited [4].

The authors, both as researchers who collect and interpret data and as scholars in training with long-term, in-depth, experience with the communities in question, hold that these shortcomings cannot be addressed without giving voice to leaders of the Asian American community. This situation was summed up eloquently by Giles Li, Director of the Boston Chinatown Neighborhood Center, during VietAID testimony [12]:

"The only reason we have any data about our [Asian American] populations at all is that community leaders have advocated for more detailed and better data for decades. The disaggregation of Asian data is not a new issue; in fact, it dates back at least to the federal government's release of the Heckler report in 1985, when Asians and Pacific Islanders were reported to have better health outcomes than all other groups. That faulty assumption was based on small samples of aggregate data, which hid the very real health disparities that different Asian populations faced. Data alone are agnostic. Data alone help us interpret and understand the truth about our communities. Data alone do not cause inequality but can help us better understand it."

If Asian Americans' access to rehabilitation assistance, employment assistance, educational options, and other supports are to be increased, if service delivery to them is to be made more culturally appropriate and effective [16], if their working and personal lives are to be improved as a result, and if their wider communities are to benefit from these improvements over time, then researchers must include them in the identification and resolution of research questions and interventions $[50,55,56]$.

In order to benefit from future research, and take advantage of funding opportunities, researchers must address the cultural and service needs of Asian Americans with disabilities, and must do so on local and regional levels. The present reliance on incomplete, inaccurate, and possibly obsolete data, compiled nationally, is inadequate. Increased local focus is necessary to tailor initiatives to the populations in question and to acknowledge the fact that Asian Americans live throughout the US. For example, only $10 \%$ of the total Asian American population lives in the western region (33\% of these in San Francisco). The majority of Asian Americans are found in California, New York, Texas, New Jersey, Hawaii, and Illinois. However, relatively isolated Asian Americans deserve the same services as those who live in concentrated, well-known communities, even if it is more difficult to locate, study, and serve these smaller populations (such as more recently, the Rohingya community in the US).

\subsection{Interpretations of Disability: Reviewing Cultural Differences across Pan-Asian Groups}

Like every other ethnic or racial group in the US, Asian Americans experience disabilities and chronic health conditions that require attention, intervention, and sustained assistance. Yet mainstream academicians, service providers, funders, and policy makers seldom understand this fact, although it is both obvious and reasonable. As previously explained, the US Census does not appropriately recognize Asian ethnic groups and cultures. However, analyses reveal a great deal of intersectional variability [37] in these groups' social and health statuses when they are accurately parsed and the data disaggregated $[50,55]$. Such variability can be decisive.

One important example of the deleterious effects of "aggregating heterogenous groups appeared when a broad coalition known as VietAid [12] testified to the Massachusetts state legislature in favor of House Bill 3361, which enables the commonwealth to support data aggregation efforts within its Asian American communities. According to this testimony, 35\% of Asian Americans "reported that they 
spoke English less than 'very well,' whereas $61.2 \%$ of Vietnamese reported higher levels of limited English proficiency".

To illustrate further, the cervical cancer rate among Asian American women generally is $8.8 \%$, but Vietnamese women have almost double that rate (14.0\%) because they often do not seek medical care for symptoms or receive screening for such conditions [14]. Still another challenge arises when researchers apply ambiguous concepts of disability, including generalized diagnostic labels, such as autism and developmental disability, to subgroups of Asian American populations. Hasnain, Cohon, Shaikh, and Shanawani [57] show that none of the Pakistani languages has an equivalent word for autism. Instead, "autism", as one example, may be linked to other, unrelated terms/descriptors or to cultural meanings in another language that can erroneously influence research findings and interpretations.

In the next sections, we (1) highlight the status of large-scale data programs; and (2) offer some ways to bridge these gaps and some research methodologies that can improve the access to services and quality of outcomes for Asian Americans with disabilities and their families.

\section{Status of Large-Scale Data Programs and Asian Americans with Disabilities}

The racial and ethnic diversity of Asian Americans tends to receive greater attention in the large-scale national data systems focused on the surveillance of census, health, or employment status. However, none of these data surveillance systems are adequate for monitoring Asian Americans with disabilities. The major datasets are limited by (1) screening protocols, which are inconsistent in their classification of Asian subgroups; and (2) chronic under sampling of Asian Americans.

Undersampling is the more pernicious limitation. Most policy-relevant research questions require one or more comparisons of subgroups; few of the large national datasets contain adequate sample sizes for stable estimations in even the most rudimentary comparisons.

There is no consensus around the definition of "disability". Nonetheless, the majority of national and international data systems have converged on the merger of two conventions: (1) condition- or impairment-based schemes, and (2) limitations in specific life function or activities. Condition-based schemes ask if the person has a particular condition or diagnosis, such as deafness.

In the second approach, identification is based on loss of function; the presence or absence of a specific condition is secondary to limitations in executing activities of daily living, engaging in work, etc. A relevant distinction might involve determining if the person has difficulty walking up or down steps or engaging in physical activities designated as "normal" (e.g., bathing without assistance). This distinction is important because it extends the definition of disability beyond diagnosis to incorporate the ability to function, either well or independently; contemporary statistical data systems employ a combination of both approaches.

Discrepancies in prevalence rates are almost entirely driven by differences in definition $[27,58]$. Here, it is important to keep in mind that survey protocols and methods are under continuous review and revision (e.g., disability coding in the National Health Interview Survey). In turn, summaries are based on recent survey protocols. Modifications, however, do not alter the core point regarding inadequate sample sizes.

Table 2 summarizes selected major national datasets most often used in disability research in terms of their definitional schemes for Asian ethnic subgroupings and their estimates of subgroup prevalence. 
Table 2. Asian Americans with Disabilities in National Datasets.

\begin{tabular}{|c|c|c|c|c|}
\hline Data System & Ethnic Identifiers & $\begin{array}{l}\text { Percent Asian in US } \\
\text { General Population }\end{array}$ & $\begin{array}{c}\text { Disability Prevalence } \\
\text { within US } \\
\text { Asian Population }\end{array}$ & $\begin{array}{c}\text { Asian Disability } \\
\text { Prevalence within US } \\
\text { General Population }\end{array}$ \\
\hline $\begin{array}{l}\text { American Community } \\
\text { Survey (2018) }\end{array}$ & $\begin{array}{c}\text { Asian Indian, Chinese, } \\
\text { Filipino, Japanese, } \\
\text { Korean, Vietnamese, } \\
\text { Other Asian }\end{array}$ & $5.6 \%$ & $6.9 \%$ & $0.39 \%$ \\
\hline $\begin{array}{c}\text { National Health } \\
\text { Interview Survey (2018) }\end{array}$ & $\begin{array}{c}\text { Asian Indian, Chinese, } \\
\text { Filipino, Japanese, } \\
\text { Korean, Vietnamese }\end{array}$ & $6.2 \%$ & $8.6 \%$ & $0.54 \%$ \\
\hline $\begin{array}{c}\text { Survey of Income \& } \\
\text { Program Participation } \\
(2014)^{\mathrm{a}}\end{array}$ & $\begin{array}{c}\text { Asian only \& queries } \\
\text { about language used at } \\
\text { home (Chinese, } \\
\text { Japanese, Korean, } \\
\text { Filipinos/a, Vietnamese }\end{array}$ & $5.4 \%$ & $11.0 \%$ & $0.63 \%$ \\
\hline
\end{tabular}

a The 2014 SIPP employs a broader mix of limitations in functions, activities of daily living, instrumental activities, cognitive, sensory or other physical impairments.

Table 2 underscores two types of variability: (1) ethnic identification across even the largest of the national data systems; and (2) the sample sizes available to analysts focused on issues related to subgroups of Asians with a disability. In sum, Tables 1 and 2 summarize the research literature showing: (1) the absence of Asians in studies of minorities; and (2) that studies tend to lump all Asians together or only focus on one subgroup (e.g., Chinese).

Examples of the latter type of study generally find more pronounced needs in suicide prevention for Asians generally [22] and in depression and PTSD among Cambodian refugees [22]. Such ethnicspecific studies indicate important research needs. Supplementary S1 and S2, which affirm these two observations, summarize the broader research literature and intersectionality. The next sections contain suggestions of ways to bridge these gaps and describe some research methodologies that can improve access to services and quality of outcomes for Asian Americans with disabilities and their families.

\section{Potential for Expanding Data Availability}

Improving the sensitivity of disability data for Asian Americans will require the resolution of several important issues. First, greater consistency in data collection systems will have to be arranged, in terms of both their ethnic taxonomies and their approaches to disability screening. The derivation of sample sizes that are useful for the analysis of subgroups will likely require oversampling. However, the oversampling of targeted groups (such as Asian Americans with a disability) is cost-prohibitive and, if undertaken, would likely not be sustainable in the current budget climate.

A second, related need is for a sustained longitudinal data effort. Asian Americans constitute a dynamic, growing, and rapidly evolving demographic; capturing trends within this demographic is arguably more important than creating point-in-time estimates. Recurring administrative service registries, for example those found in state health systems or national programs, such as Medicaid, are important sources of disability data but are limited to individuals and families who are already connected to the system. Increasing the provision of service data would begin to address the critical issue of unmet needs among Asian American subgroups.

Third, researchers need not rely on random samplings of households or geographic units. It is particularly important to keep this in mind because logistical challenges are abound in the development of demographic profiles of low-prevalence (rare) populations because conventional sampling procedures, while addressing representativeness for the overall sample, do not allow reliable disaggregation. As illustrated in Table 2, Asian Americans with disabilities can be considered a "rare population" [59].

Alternative strategies include the use of cluster sampling and screening within the cluster, or the selection of clusters that contain a high proportion of targets (e.g., community groups). Disproportionate stratified sampling can also work if researchers have valid reasons to believe that higher rates are associated with certain demographic strata; other variations on sampling tactics are available as 
well [60]. More recent innovations such as multistage samplings through service providers or cultural centers rather than random sampling units [61] hold promise as well.

In nations with poor or nonexistent statistical infrastructure, researchers have used Demographic Surveillance Sites (DSS) effectively for large-scale studies [62]. When researchers consider these and other conventional alternative strategies, however, they must remember that their efficiency may not be adequate from a resource perspective if (1) the target population is not randomly distributed across demographic strata such as age or income levels; and (2) sampling clusters associated with Asian Americans (e.g., community centers, health associations, and religious groups) are limited and highly heterogeneous.

The easy but unrealistic solution would be to call for the commitment of significant resources for the development of large, comprehensive, technically integrated data profiles. This outcome is highly unlikely, however, in an era of shrinking public resources for general data surveillance. Furthermore, because data is collected for specific purposes that in turn dictates methodology and sampling approaches, no single approach can be optimal.

We suggest that researchers, program evaluators, and analysts should begin to prioritize the interrogation of Asian American disability diversity within their research and evaluation efforts and to incorporate more sophisticated taxonomies of Asian subgroups. Such prioritization could mean, for example, sampling a homogeneous ethnic group rather than a broadly (and loosely) defined "Asian" sample. Because the immediate need is a more nuanced understanding of the diversity of issues within the larger population, the analytic task in the field is to integrate significantly different efforts into a compiled, complementary data portrait. Engaging with this task would be an imperfect but important first step in better understanding the Asian American population. The next section presents some of the challenges of conducting research on Asian Americans who are living with disability, and some solutions.

\section{Methodological Challenges and Solutions in Conducting Research}

A careful study of the literature revealed that many of studies use intact groups, an important design weakness that results in unknown sampling biases. In field research studies with this shortcoming, differences between Asians and Whites are often attributed to racial/ethnic status, while collateral influences, such as level of education or other biopsychosocial determinants, are not controlled. Conversely, biased samples may obscure substantial differences that would otherwise be apparent if researchers employed comparable samples via randomization or statistical controls. It is precisely these uncredited, unrecognized differences, however, that are most important for the development and delivery of effective care systems.

Another important facet of minority research concerns within-group differences, which are often larger than between-group differences [63]. Immigration status is important as well. A first-generation immigrant has a different experience and milieu than an established, fourth-generation Asian American. Across cultural groups, researchers should use two key criteria for comparison [64]. First, measures used in research must have the same or highly similar meanings across all languages to ensure a level of linguistic accuracy. Native speakers or trained, certified translators of all the relevant languages should translate where necessary. Second, the reliability of test scores across populations depends on metric equivalence. For example, based on cultural frameworks alone, a Buddhist may respond differently from a Hindu when asked by a doctor to rate physical pain on a scale of 1 to 10 . To counteract this type of issue, multiple scales should be used.

Cultural bias is another mitigating factor. Researchers and providers alike must eliminate bias to the greatest extent possible, whether it is real or perceived. The latter reduces community participation, which is an essential feature of minority-based projects. Ideally, community-based research employs community outreach while also allowing community members to be true stakeholders by participating in the research beyond simple data collection. Local researchers who are in sync with a particular community should involve themselves as much as possible and should seek insider insight from 
participants. In order to effectively work with Asian Americans, research processes must exhibit validity in terms of cultural context. This means that researchers must develop studies and interventions with the explicit goals of not only giving voice to Asian American groups, but also recognizing and seeking to enhance the strengths of these populations, while reaching a balance of power among all stakeholders [65].

A related cultural phenomenon is the Asian American perception of disability. For example, their perceptions of what disability means can affect the way they respond to interviewers, as well as how they complete forms and surveys. Even when researchers use specific, functional terminology, there is ample room for misinterpretation. Therefore, it is essential that researchers have accurate translations and that the implied meanings of words and linguistic styles are understandable to study participants. Unless researchers conduct studies in culturally relevant ways that extend beyond linguistic accuracy, a variety of factors can threaten rigor; these include insufficient descriptions and inconsistent analyses. In addition, false negatives are common due to disability stigma but would be less problematic if they occurred randomly; however, research strongly suggests that major variations in disability stigma exist across ethnic groups and depend on the degree of acculturation and place of birth $[66,67]$. Such misinterpretations likely result in significant variability within derived disability rates [68]. In addition to issues of definition and taxonomy, other methodological issues include the need to incorporate cultural and linguistic competency in all stages of development and implementation. Competency potentially affects all stages of the research process, such as sample recruitment and the validity of the study measures.

A fourth challenge is the lack of information regarding the ways in which particular recruitment methods affect the selection characteristics of Asian American participants. Among studies that focus on this population, many tend to recruit participants through ethnic venues by using simple, familiar sampling strategies (e.g., snowball method). Recently, a few researchers have employed a random sampling strategy that utilizes mail or telephone survey methods. In one study using a mail survey, an offer of monetary incentives instead of the offer of a small gift improved the response rates among the target group-employed Chinese and Korean American men. A convenience sampling method brought in more students who were younger, but the differences in yields were not significant [69].

\section{Proposal of CBPR as an Alterative}

A potentially important tool in further developing the body of research is participatory approaches to conducting research. As it happens, these approaches have been shown to aid the development of best practices in the area of Asian American disability research and policy development $[50,70]$. Better known as community-based participatory research (CBPR), participatory research involves key stakeholders from the community and places high value on sharing methodological knowledge with participants. Not only does such an approach recognize and draw on the strengths and resources of a community, it also provides a long-term mechanism for creating sustainable change and building capacity. Most studies that employ CBPR as a research tool focus on specific ethnic communities, but it is particularly useful for studying Asian Americans because of their tendency to live in ethnic enclaves.

Because of the extraordinary diversity, even large-sample studies will inadequately represent the larger population. Not surprisingly, most of the successful empirical studies of Asian Americans with a disability are smaller scale or involve local communities. For example, Chung [28] worked with the Malden (Massachusetts) Asian Disability Advocacy Coalition and the Great Wall Center to develop a needs-based assessment of disability and health.

National-level projects would do well to follow this example. Tanjasiri et al. [71], who conducted a study on breast cancer in the Hmong community, recruited participants through nonprobability sampling, but were unable to establish whether this particular group of Hmong women (age 40 years and older) differed from Hmong with the same characteristics in the general population. Still, their recruitment procedures, which drew on existing networks and opportunities, demonstrated 
a culture-friendly strategy for approaching smaller refugee communities where trust-building is necessary.

Asian-specific research is conducted most often in California, followed by New York City, Massachusetts, Hawaii, Seattle-Tacoma, Houston, Philadelphia, District of Columbia, and Chicago. The methods of community engagement most frequently used by Asian-centered projects include consulting with and acquiring feedback from community representatives as well as partnering with CBOs, Asians with disabilities, and other stakeholders. A routine feature of the lead author's own research is the establishment of formal advisory boards composed of community members-an irreplaceably helpful resource. Board member involvement extends to setting the research question(s) and monitoring outcomes throughout the life of a project to giving input on the set-up of analysis procedures. In many cases, the results of research projects with high-level community involvement tend to show higher levels of accuracy, utility, and replicability.

\section{Conclusions}

This conceptual paper, which is not a systematic review, presents an overview of the current state of the literature in Asian American disability and mental health studies. By conducting a structured literature search rather than a systemic analysis, conclusions made from this synthesis results are more qualitative in nature and broader in scope. The search process was intentionally wide-ranging, in order to uncover all sorts of methodological inadequacies in research studies pertaining to disability among Asian population groups in the US or English-speaking countries. Critical analyses of the included papers' database resources and analytical results, including an evaluation of robust participant recruitment processes and an assessment of the statistical significance of the study findings, were not performed. Despite this limited approach, the literature review strategy revealed common limitations. Foremost among these was not accounting for Asian subgroup diversity, taxonomical inconsistencies, and population sampling issues. The result is an absence of specific policy and practice-relevant information on Asian Americans with a disability.

This examination of the issues and implications of ignoring the diversity of the Asian American population in disability research underscores the absence of culturally sensitive research and the impediments and barriers to effective methodologies for studying Asian Americans with disabilities or other racial-ethnic groups. While sparse, the review revealed a significant and consistent gap between mainstream disability services and the needs of Asian Americans with disabilities. To better understand this gap, and other needs still unknown, we argue that disability researchers must strive to incorporate more refined taxonomies of Asian subgroups. As researchers evaluate impediments to their studies on these groups, the alternative methodologies presented herein can help them better address heterogeneity in ways that are responsive, inclusive, and productive.

Finally, researchers must employ culturally appropriate measures by using sampling processes that adequately capture diversity of Asian American populations and by honoring worldviews and cultural histories of these populations. Researchers must also explore ways to include families and communities in studies of Asian American individuals with disabilities, specifically by building sustainable, community-based partnerships that allow stakeholders to contribute to the successive phases of research, development, and implementation [72]. These approaches, when implemented, are likely to add to the literature as well as to positively affect policy and funding for this overlooked, understudied demographic.

\section{Limitations and Future Steps}

The following future steps are based on limitations in the current literature. First, researchers should be careful not to generalize about disability issues across and within Asian groups; at a minimum, they should openly acknowledge the limitations of the general label. Second, whenever possible, research should disaggregate Asian data by ethnic group. Third, we recommend that researchers employ alternative sampling methodologies designed for "rare" populations. Fourth, researchers 
should focus on specific subethnic groups if sampling resources are limited. Fifth, researchers should collect data on immigration and refugee status whenever appropriate and possible.

Finally, researchers should adopt multi-faceted approaches in local community capacity-building studies. Although all types of research approaches may be necessary for developing an accurate portrait of Asian Americans with disabilities, CBPR is uniquely positioned to overcome many of the impediments to research with this population. The economics of big research suggest that major national initiatives focused on better understanding variations within the larger Asian community are unlikely and that if funded they would not be sustainable. Most inquiries, therefore, will likely involve local studies or evaluations. CBPR fits well with a culturally tailored integrative approach through its use of partnerships, procedural focus on gatekeepers, and emphasis on co-learning relationships and sustainability.

There are no simple solutions to the lack of comprehensive data, and we suggest that the near-term need is for researchers to abandon the "Asian" subgroup in favor of more nuanced understandings of smaller groups. This will require in turn, the nuanced use of data drawn from complementary but not necessarily equivalent data portraits using different samples, methodologies, and disciplinary lenses. While imperfect it is the important first step in understanding the diversity of what is currently considered "Asian" in the context of disability.

Supplementary Materials: The following are available online at http://www.mdpi.com/2075-4698/10/3/58/s1, Supplementary Material 1: Asian Disability Methodology Included Asians Articles, and Supplementary Material 2: Minority Research Publications without Asians.

Author Contributions: Conceptualization, R.H., G.T.F. and J.E.C.; methodology, R.H. and G.T.F.; validation, J.E.C., T.T.T.B. and S.K.; formal analysis, R.H., G.T.F., J.E.C. and T.T.T.B.; investigation, R.H. and G.T.F., resources, J.E.C., T.T.T.B. and S.K.; data curation, R.H., J.E.C., T.T.T.B. and S.K.; writing-original draft preparation R.H., G.T.F. and J.E.C.; writing — review and editing, R.H., G.T.F. and J.E.C.; visualization, T.T.T.B.; supervision, R.H.; project administration, R.H. All authors have read and agreed to the published version of the manuscript.

Funding: This research received no external funding.

Acknowledgments: The authors like to acknowledge the important work that has been conducted and/or supported by fellow researchers and allies on aspects of this topic.

Conflicts of Interest: The authors declare no conflict of interest.

\section{References}

1. US Census Bureau Asian-American and Pacific Islander Heritage Month: May 2019. 2019. Available online: https://www.census.gov/newsroom/facts-for-features/2019/asian-american-pacific-islander.html (accessed on 15 June 2020).

2. Unted Nations. Convention on the Rights of Persons with Disabilities; United Nations: New York, NY, USA, 2006. Available online: http://www.un.org/disabilities/ (accessed on 10 June 2020).

3. Montalto, N.; Hasnain, R. Systems Change for Greater Cultural Competence in the Pennsylvania Disability Service and Support Sector. A Report to the Pennsylvania Developmental Disabilities Council; Diversity Dynamics, LLC: Cranford, NJ, USA, 2011.

4. Hsueh-Fen, S.; Hsu, M.-T.; Clark, L. Conceptualizing and critiquing culture in health research. J. Transcult. Nurs. 2004, 15, 269-277. [CrossRef]

5. United Nations. Asia and the Pacific; United Nations: New York, NY, USA. Available online: https: //www.un.org/en/sections/where-we-work/asia-and-pacific/index.html (accessed on 18 June 2020).

6. Trimble, J.E.; Fisher, C.B. The Handbook of Ethical Research with Ethnocultural Populations and Communities; Sage Publications: Thousand Oaks, CA, USA, 2006.

7. US Department of Health and Human Services Office of Minority Health (OMH). Profile: Asian Americans; US Department of Health and Human Services: Rockville, MD, USA, 2019.

8. Passel, J.; Cohn, D. US Population Projections: 2005-2050; Pew Research Center: Washington, DC, USA, 2008. Available online: https://www.pewresearch.org/hispanic/2008/02/11/us-population-projections-2005-2050/ (accessed on 18 June 2020). 
9. Palafox, N.A.; Buenconsejo-Lum, L.; Riklon, S.; Waitzfelder, B. Improving health outcomes in diverse populations: Competency in cross-cultural research with indigenous Pacific Islander populations. Ethn. Health 2002, 7, 279-285. [CrossRef] [PubMed]

10. López, G.; Ruiz, N.G.; Patten, E. Key Facts about Asian Americans, a Diverse and Growing Population; Pew Research Center: Washington, DC, USA, 2017. Available online: https://www.pewresearch.org/fact-tank/2017/09/08/ key-facts-about-asian-americans/ (accessed on 8 September 2017).

11. Ramakrishnan, K.; Ahmad, F. Language Diversity and English Proficiency: Part of the "State of Asian Americans and Pacific Islanders" Series; Center for American Progress: Washington, DC, USA, 2014. Available online: https://cdn.americanprogress.org/wp-content/uploads/2014/04/AAPI-LanguageAccess1.pdf (accessed on 21 June 2020).

12. VietAID. Asian American Data Aggregation at House Bill 3361 Hearing. 2018. Available online: https: //www.vietaid.org/2018/01/30/asian-american-data-disaggregation-at-house-bill-3361-hearing/ (accessed on 15 June 2020).

13. Fujiura, G.T.; Drazen, C. "Ways of seeing" in race and disability research. In Race, Culture and Disability: Rehabilitation Science and Practice; Balcazar, E.F., Suarez-Balcazar, Y.S., Taylor-Ritzler, T., Keys, C.B., Eds.; Jones and Bartlett: Sudbury, MA, USA, 2009; pp. 15-32.

14. Ghosh, C. Asian American health research: Baseline data and funding. In Asian American Communities and Health: Context, Research, Policy and Action; Trinh-Shevrin, N.S., Islam, M.J., Rey, C., Eds.; Jossey-Bass: San Francisco, CA, USA, 2009; pp. 73-103.

15. Mirza, M.; Hasnain, R.; Duke, B.K. Fostering community-academic partnerships to promote employment opportunities for refugees with disabilities: Accomplishments, dilemmas, and deliberations. Societies 2018, 8, 86. [CrossRef]

16. Hasnain, R.; Leung, P. Cross-cultural issues in the vocational rehabilitation system for Asian-Pacific Americans with disabilities. In Culture, Race, and Ethnicity; Balcazar, F., Balcazar, Y., Keys, C., Taylor-Ritzler, T., Eds.; Jones \& Bartlett Publishers: Reading, MA, USA, 2010.

17. Ngo-Metzger, Q.; Kaplan, S.H.; Sorkin, D.H.; Clarridge, B.R.; Phillips, R.S. Surveying minorities with limited-English proficiency: Does data collection method affect data quality among Asian Americans? Med. Care 2004, 42, 893-900. [CrossRef]

18. Akande, A.O. Factors Affecting Vocational Goal Acquisition of Female Immigrant Clients: Rehabilitation Counselor Perspectives. J. Rehabil. 2017, 83, 11-22.

19. Millner, U.C.; Kim, M. Perspectives on work and work-related challenges among Asian Americans with psychiatric disabilities. Asian Am. J. Psychol. 2017, 8, 177-189. [CrossRef]

20. Mirza, M.; Heinemann, A.W. Service needs and service gaps among refugees with disabilities resettled in the United States. Disabil. Rehabil. 2012, 34, 542-552. [CrossRef]

21. Yan, K.K.; Accordino, M.P.; Boutin, D.L.; Wilson, K.B. Disability and the Asian culture. J. Appl. Rehabil. Couns. 2014, 45, 4-8. [CrossRef]

22. Applied Research Center \& the National Council of Asian Pacific Americans (NCAPA). Best Practices: Researching Asian Americans, Native Hawaiian and Pacific Islanders. 2013. Available online: http://depts. washington.edu/sphnet/wp-content/uploads/2013/01/BestPracticesAANHPI.pdf (accessed on 1 June 2020).

23. Ogilvie, L.D.; Burgess-Pinto, E.; Caufield, C. Challenges and approaches to newcomer health research. J. Transcult. Nurs. 2008, 19, 64-73. Available online: https://journals.sagepub.com/doi/10.1177/ 1043659607309142 (accessed on 1 June 2020). [CrossRef]

24. White House Executive Order 13125. White House Initiative on Asian Americans and Pacific Islanders. 1999. Available online: https://clintonwhitehouse4.archives.gov/WH/New/APA/whaapi.html (accessed on 24 June 2020).

25. White House Executive Order 13515 (posted June 7, 1999). The President Executive Order 13515-Increasing Participation of Asian Americans and Pacific Islanders in Federal Programs. Available online: https: //www.hsdl.org/?abstract\&did=799535 (accessed on 23 June 2020).

26. Joo, N.; Reeves, R.V.; Rodrigue, E. Asian-American success and the pitfalls of generalization; Brookings Institute: Washington, DC, USA, 2016. Available online: https://www.brookings.edu/research/asian-american-successand-the-pitfalls-of-generalization/ (accessed on 23 June 2020).

27. Yang, H.; Leung, P.; Wang, J.; Shim, N. Asian Pacific Americans: The need for ethnicity-specific disability and rehabilitation data. J. Disabil. Polices Stud. 1996, 7, 33-53. [CrossRef] 
28. Chung, T.L. Asian Health and Disability Issues in Malden, Massachusetts; The Malden Asian Disability Advocacy Coalition: Malden, MA, USA, 2005.

29. Pew Research Center. The Rise of Asian Americans; Social and Demographic Trends: Washington, DC, USA, 2013; Available online: https://www.pewsocialtrends.org/2012/06/19/the-rise-of-asian-americans/ (accessed on 20 June 2020).

30. Hing, J. Asian Americans respond to Pew: We're not your model minority. ColorLines: News for Action, 21 June 2012. Available online: https://www.colorlines.com/articles/asian-americans-respond-pew-werenot-your-model-minority (accessed on 1 June 2020).

31. Stodden, R.A.; Stodden, N.J.; Kim-Rupnow, W.S.; Thai, N.D.; Galloway, L.M. Providing effective support services for culturally and linguistically diverse persons with disabilities: Challenges and recommendations. J. Vocat. Rehabil. 2003, 18, 177-189.

32. Kim-Rupnow, W.S.; Park, H.C.; Starbuck, D.E. Status overview of vocational rehabilitation services for Asian Americans and Pacific Islanders with disabilities. J. Vocat. Rehabil. 2005, 23, 21-32.

33. Southwest Educational Development Laboratory Disability research for all. Designing and conducting research with diverse consumer groups: Implications and considerations. Res. Exch. 2001, 6, 3-8.

34. Erickson, W.; Lee, C.; von Schrader, S. 2017 Disability Status Report: United States; Cornell University Yang-Tan Institute on Employment and Disability (YTI): Ithaca, NY, USA, 2019.

35. President's Advisory Commission on Asian Americans and Pacific Islanders. Asian Americans and Pacific Islanders: A People Looking forward: Action for access and Partnerships in the 21st Century: Interim Report to the President and the Nation; The Commission: Washington, DC, USA, 2001.

36. De Souza, L.R.; Fuller-Thomson, E. Acculturation and disability rates among Filipino-Americans. J. Immigr. Minority Health 2013, 15, 462-471. [CrossRef] [PubMed]

37. Hodgkins, S.L.; Mereish, E.H. The intersectional invisibility of race and disability status: An exploratory study of health and discrimination facing Asian Americans with disabilities. Ethn. Inequalities Health Soc. Care 2012. [CrossRef]

38. Jang, Y.; Chiriboga, D.A.; Allen, J.Y.; Kwak, J.; Haley, W.E. Willingness of older Korean-American Adults to use hospice. J. Am. Geriatr. Soc. 2010, 58, 352-356. [CrossRef]

39. Jang, Y.; Kim, G.; Chiriboga, D.A. Gender differences in depressive symptoms among older Korean American immigrants. Soc. Work Public Health 2011, 26, 96-109. [CrossRef]

40. Min, J.W. Preference for long-term care arrangement and its correlates for older Korean Americans. J. Aging Health 2005, 17, 363-395. [CrossRef]

41. Poon-McBrayer, K.F.; García, S.B. Profiles of Asian American students with LD at initial referral, assessment, and placement in special education. J. Learn. Disabil. 2000, 33, 61-71. [CrossRef]

42. Parette, P.; Chuang, S.J.L.; Blake Huer, M. First-generation Chinese American families' attitudes regarding disabilities and educational interventions. Focus Autism Other Dev. Disabil. 2004, 19, 114-123. [CrossRef]

43. Wang, H.T.; Casillas, N. Asian American parents' experiences of raising children with autism: Multicultural family perspective. J. Asian Afr. Stud. 2013, 48, 594-606. [CrossRef]

44. Gabel, S. South Asian Indian cultural orientations toward mental retardation. Ment. Retard. 2004, 42, 12-25. [CrossRef]

45. Baker, D.L.; Miller, E.; Dang, M.T.; Yaangh, C.S.; Hansen, R.L. Developing culturally responsive approaches with Southeast Asian American families experiencing developmental disabilities. Pediatrics 2010, 126 (Suppl. 3), S146-S150. [CrossRef] [PubMed]

46. Kwong, K.; Chung, H.; Cheal, K.; Chou, J.C.; Chen, T. Disability beliefs and help-seeking behavior of depressed Chinese-American patients in a primary care setting. J. Soc. Work Disabil. Rehabil. 2012, 11, 81-99. [CrossRef] [PubMed]

47. Asian Pacific American Legal Center and Asian American Justice Center. A Community of Contrasts: Asian Americans in the United States 2011, Asian American Center for Advancing Justice. 2011. Available online: http://advancingjusticela.org/system/files/ENTERED_Community_of_Contrasts_2011.pdf (accessed on 10 May 2020).

48. Hoeffel, E.; Rastogi, S.; Kim, M.; Shahid, H. The Asian Population: 2010 Census Briefs. 2012. Available online: https://www.census.gov/prod/cen2010/briefs/c2010br-11.pdf (accessed on 18 June 2020).

49. Amzel, A.; Ghosh, C. National newspaper coverage of minority health disparities. J. Natl. Med. Assoc. 2007, 99, 1120-1125. 
50. Chin, J.L.; Mio, J.S.; Iwamasa, G.Y. Ethical conduct of research with Asian and Pacific Islander American populations. In The Handbook of Ethnical Research with Ethnocultural Populations and Communities; Trimble, J.E., Fisher, C.E., Eds.; Sage: Thousand Oaks, CA, USA, 2006; pp. 117-135. [CrossRef]

51. Asian American Psychological Association Leadership Fellows Program. Suicide among Asian Americans. 2014. Available online: https://www.apa.org/pi/oema/resources/ethnicity-health/asian-american/suicide-factsheet.pdf (accessed on 28 June 2020).

52. Akobirshoev, I.; Mitra, M.; Parish, S.L.; Moore Simas, T.A.; Dembo, R.; Ncube, C.N. Racial and ethnic disparities in birth outcomes and labour and delivery-related charges among women with intellectual and developmental disabilities. J. Intellect. Disabil. Res. 2019, 63, 313-326. [CrossRef]

53. Islam, N.S.; Trinh-Shevrin, C.; Rey, M.J. Toward a contextual understanding of Asian American health. In Asian American Communities and Health: Context, Research, Policy and Action; Trinh-Shevrin, C., Islam, N.S., Rey, M.J., Eds.; Jossey-Bass: San Francisco, CA, USA, 2009; pp. 3-22.

54. Museus, S.D.; Kiang, P.N. Deconstructing the model minority myth and how it contributes to the invisible minority reality in higher education research. New Dir. Inst. Res. 2009, 2009, 5-15. [CrossRef]

55. Srinivasan, S.; Guillermo, T. Toward improved health: Disaggregating Asian American and native Hawaiian/Pacific Islander data. Am. J. Public Health 2000, 90, 1731-1734. [CrossRef]

56. Hampton, N.Z. Asian Americans with Disabilities: Access to education, health care, and rehabilitation services. In Asian Americans: Vulnerable Populations, Model Interventions, and Clarifying Agendas; Zhan, L., Ed.; Johnes and Bartlett Publishers: Sudbury, MA, USA, 2003; pp. 69-88.

57. Hasnain, R.; Cohon, L.; Shaikh, L.C.; Shanawani, H. Disability and the Muslim perspective: An introduction for rehabilitation and health care providers. In Center for International Rehabilitation Research Information and Exchange (CIRRIE); 2008. Available online: https://digitalcommons.ilr.cornell.edu/gladnetcollect/460/index. html (accessed on 30 March 2020).

58. Fujiura, G.T.; Rutkowski-Kmitta, V. Counting disability. In Handbook of Disability Studies; Albrecht, L.G., Seelman, K.D., Bury, M., Eds.; Sage Publications: Thousand Oaks, CA, USA, 2001; pp. 69-96.

59. Sudman, S. Applied sampling. In Handbook of Survey Research; Rossi, P.H., Wright, J.D., Anderson, A.B., Eds.; Academic Press: New York, NY, USA, 1976; pp. 145-194.

60. Kish, L. Survey Sampling; John Wiley \& Sons: New York, NY, USA, 1965.

61. Frankel, M.R.; Shapiro, M.F.; Duan, N.; Morton, S.C.; Berry, S.H.; Brown, J.A.; Burnam, M.A.; Cohn, S.E.; Goldman, D.P.; McCaffrey, D.F.; et al. National probability samples in studies of low-prevalence diseases. Part II: Designing and implementing the HIV cost and services utilization study sample. Health Serv. Res. 1999, 34 Pt 1, 969-992.

62. Fottrell, E.; Byass, P. Population survey sampling methods in a rural African setting: Measuring mortality. Popul. Health Metr. 2008, 6, 1-11. [CrossRef]

63. Sue, D.W.; Sue, D. Counseling the Culturally Diverse: Theory and Practice; John Wiley \& Sons, Inc.: San Francisco, CA, USA, 2003.

64. Sue, S.; Kurasaki, K.S.; Srinivasan, S. Ethnicity, gender, and cross-cultural issues in clinical research. In Handbook of Research Methods in Clinical Psychology, 2nd ed.; Kendall, P.C., Butcher, J.N., Holmbeck, G.N., Eds.; John Wiley \& Sons, Inc.: Hoboken, NJ, USA, 1999; pp. 54-71.

65. Paloma, V.; Herrera, I.M.; García-Ramírez, M. Psychopolitical validation of health promotion research for migrant populations: Conceptualising well-being among Andalusian Moroccan immigrants. Int. J. Migr. Healthsoc. Care 2009, 5, 25-33. [CrossRef]

66. Chen, R.K.; Brodwin, M.G.; Cardoso, E.; Chan, F. Attitudes toward people with disabilities in the social context of dating and marriage: A comparison of American, Taiwanese, and Singaporean students. J. Rehabil. $2002,68,5-11$.

67. Saetermoe, C.L.; Scattone, D.; Kim, K.H. Ethnicity and the stigma of disabilities. Psychol. Health 2001, 16, 699-713. [CrossRef]

68. Suh, E.E.; Kagan, S.; Strumpf, N. Cultural competence in qualitative interview methods with Asian immigrants. J. Transcult. Nurs. 2009, 20, 194-201. [CrossRef] [PubMed]

69. Lee, S.K.; Cheng, Y.Y. Reaching Asian Americans: Sampling strategies and incentives. J. Immigr. Minority Health 2006, 8, 245-250. [CrossRef] [PubMed] 
70. Tandon, S.D.; Kwon, S.C. Community-based participatory research. In Asian American Communities and Health: Context, Research, Policy and Action; Trinh-Shevrin, C., Islam, N.S., Rey, M.J., Eds.; Jossey-Bass: San Francisco, CA, USA, 2009; pp. 464-503.

71. Tanjasiri, S.P.; Kagawa-Singer, M.; Foo, M.A.; Chao, M.; Linayao-Putman, I.; Nguyen, J.; Pirumyan, G.; Valdez, A. Designing culturally and linguistically appropriate health interventions: The "Life Is Precious" Hmong breast cancer study. Health Educ. Behav. Off. Publ. Soc. Public Health Educ. 2007, 34, 140-153. [CrossRef]

72. Lindenberg, C.S.; Solorzano, R.M.; Vilaro, F.M.; Westbrook, L.O. Challenges and strategies for conducting intervention research with culturally diverse populations. J. Transcult. Nurs. 2001, 12, 132-139. [CrossRef]

C 2020 by the authors. Licensee MDPI, Basel, Switzerland. This article is an open access article distributed under the terms and conditions of the Creative Commons Attribution (CC BY) license (http://creativecommons.org/licenses/by/4.0/). 